\title{
HARMONIC FUNCTIONS AND MASS CANCELLATION ${ }^{1}$ BY \\ J. R. BAXTER
}

\begin{abstract}
If a function on an open set in $\mathbf{R}^{n}$ has the mean value property for one ball at each point of the domain, the function will be said to possess the restricted mean value property. (The ordinary or unrestricted mean value property requires that the mean value property hold for every ball in the domain.) We specify the single ball at each point $x$ by its radius $\delta(x)$, a function of $x$. Under appropriate conditions on $\delta$ and the function, the restricted mean value property implies that the function is harmonic, giving a converse to the mean value theorem (see references). In the present paper a converse to the mean value theorem is proved, in which the function $\delta$ is well behaved, but the function is only required to be nonnegative. A converse theorem for more general means than averages over balls is also obtained. These results extend theorems of D. Heath, W. Veech, and the author (see references). Some connections are also pointed out between converse mean value theorems and mass cancellation.
\end{abstract}

1. Introduction. If a function satisfies the mean value property at every point of a region, then the function is harmonic in the region. This is a familiar converse to Gauss's mean value theorem. If the function satisfies only a restricted mean value property at each point, namely that there should be one ball at each point for which the mean over the ball equals the value at the point, then again under some conditions the function can be shown to be harmonic. In the present paper (Theorem 2) a result of this sort is proved. The proof involves a mass cancellation argument that has some independent interest, and this aspect of the problem is explored in $\$ 2$.

Many previous converses to the mean value theorem have been given. Results that seem closest to the present theorem are found in [2] (generalizing a theorem in [1]), [7], [11], and [12]. We shall state the result in [12].

Let $D$ be an open subset of $\mathbf{R}^{l}$. For any $x$ in $D$, and any $r \geqslant 0$, let $B(x, r)$ denote the open ball with centre $x$ and radius $r$. Let $d$ be a fixed function on $D$, with $d(x)$ less than or equal to the distance of $x$ from $D^{c}$, such that $d>0$ and $|d(x)-d(y)| \leqslant|x-y|$ for all $x$ and $y$ in $D$. In [12], Veech proved the following:

Theorem 1. Let $\delta: D \rightarrow \mathbf{R}$ be such that there exists $\alpha>0$ with $\alpha d \leqslant \delta \leqslant(1$

Received by the editors April 22, 1977.

AMS (MOS) subject classifications (1970). Primary 60J05, 60J65; Secondary 31B05.

Key words and phrases. Restricted mean value, invariant function, Brownian motion.

${ }^{1}$ This paper was supported in part by NSF Grant MCS 74-05786 A02. 
- $\alpha) d$ on $D$. Let $D$ be a bounded Lipschitz domain. Let $h$ be a nonnegative, finite, Lebesgue measurable function such that for each $x$ in $D, h(x)$ equals the mean value of $h$ over $B(x, \delta(x))$. Then $h$ is harmonic.

In the present paper we shall generalize Theorem 1 by removing the restriction on $D$, and by allowing other types of means besides averages over balls. This result is stated below as Theorem 2 and proved in $\$ 4$. The method of proof is essentially that used by Heath in [7], and appears quite different from the proof in [11] and [12].

Before stating Theorem 2, some notation is needed. Let $m$ denote Lebesgue measure on $\mathbf{R}^{l}$, let $m_{x}$ denote the unit point mass at $x$. Let $\theta: \mathbf{R}^{l} \rightarrow \mathbf{R}$ denote the Newtonian potential kernel, so that $\theta(x)=-|x|$ in $\mathbf{R}^{1}, \theta(x)=-\log |x|$ in $\mathbf{R}^{2}$, and $\theta(x)=|x|^{-l+2}$ in $\mathbf{R}^{\prime}, l \geqslant 3$. For any positive measure $\mu$, let Pot $\mu(y)=\int \theta(y-z) \mu(d z)$. If $\mu$ is bounded and has bounded support then Pot $\mu$ exists as a finite number or $\infty$, and Pot $\mu$ is finite $m$-a.e.

Now let $\varphi>0$ be a Borel function on $\mathbf{R}^{l} \times \mathbf{R}^{l}$, such that

$$
\int \varphi(x, y) m(d y)=1 \text { for all } x \text { in } D,
$$

and let there exist constants $\alpha, \beta, \gamma>0$ such that for all $x, y$ in $D$,

$$
\beta f_{x}(y)<\varphi(x, y)<\gamma g_{x}(y),
$$

where $f_{x}$ is the indicator function of $B(x, \alpha d(x))$ divided by the volume of $B(x, \alpha d(x))$, and $g_{x}$ is the indicator function of $B(x,(1-\alpha) d(x))$ divided by the volume of $B(x,(1-\alpha) d(x))$.

Let $\mu_{x}$ denote the probability measure that has density $\varphi(x, \cdot)$ with respect to $m . \varphi$ will be assumed to be such that:

$$
\text { Pot } m_{x}>\text { Pot } \mu_{x} \text { on } \mathbf{R}^{l} \text {. }
$$

Conditions (1.2) and (1.3) easily imply that Pot $m_{x}$ and Pot $\mu_{x}$ must be equal outside $B(x,(1-\alpha) d(x))$ (cf. [3, Lemma 1, Corollary 2]) and hence, by Green's theorem, that

$$
h(x)=\int h d \mu_{x} \text { for any harmonic function } h \text { on } D .
$$

The converse to (1.4) is given as:

THEOREM 2. Let (1.1)-(1.3) hold. Let $h$ be a nonnegative, finite, Lebesgue measurable function such that for each $x$ in $D, h(x)=\int h d \mu_{x}$. Then $h$ is harmonic.

Theorem 1 easily follows from Theorem 2 if the function $\delta$ of Theorem 1 is Borel. The reduction of Theorem 1 (and other similar theorems) to the case in which $\delta$ is Borel was proved by Veech in [11].

The referee mentions that A. Ancona has shown how to modify the 
arguments in [12] to obtain Theorem 1 without assuming $\partial D$ is smooth.

2. General remarks on cancellation. In this section a form of mass cancellation is defined (Properties 2 and 4), and the connection with the converse mean value theorem is shown (Theorems 4 and 5). These results will not be required in $\$ 4$, but should motivate the approach used there. Similar results are given in Veech $[13, \S \S 9$ and 10] from a different point of view.

Let $\left(Y_{n}\right)$ be a discrete-time Markov process with operator $P$. Let $\mu$ and $\lambda$ be probability measures on the state space. Suppose there exists a randomized stopping time $\tau$ such that $Y_{\tau}$ has distribution $\lambda$ when $X_{0}$ has distribution $\mu$. Then we write $\mu \rightarrow \lambda$.

Definition 1. Let $\mu$ and $\nu$ be probability measures on the state space. If there exists $\lambda$ such that $\mu \rightarrow \lambda$ and $\nu \rightarrow \lambda$, then we write $\mu \sim \nu$, and say that $\mu$ and $\nu$ can be asymptotically cancelled.

It should be noted that under some conditions this form of mass cancellation is identical with that considered in the limit theorem of Orey [9].

TheOREM 3. Let $P$ be a Markov operator such that $\mu P^{n}-\mu P^{n+1} \rightarrow 0$ in total variation norm as $n \rightarrow \infty$, for all probability measures $\mu$. Then $\mu \sim \nu$ if and only if $(\mu-\nu) P^{n} \rightarrow 0$ in total variation norm as $n \rightarrow \infty$.

Proof. Let $\mu \sim \nu$ hold. We must show $(\mu-\nu) P^{n} \rightarrow 0$. Since $\mu \rightarrow \lambda$ and $\nu \rightarrow \lambda$ for some $\lambda$, we may assume $\mu \rightarrow \nu$ by a stopping time $\tau$. Let $\mu_{k}$ be the distribution of $Y_{\tau \wedge k}$. It is clearly enough to show that $\left(\mu-\mu_{k}\right) P^{n} \rightarrow 0$ as $n \rightarrow \infty$ for each $k$, or that $\left(\mu_{k}-\mu_{k+1}\right) P^{n} \rightarrow 0$ as $n \rightarrow \infty$ for each $k$. Hence it is really enough to consider the case $\mu \rightarrow \nu$ by $\tau$ with $0<\tau<1$. By the strong Markov property we may then reduce the problem to the case that $\tau \equiv 1$, in which case the result follows by assumption.

Conversely, let $(\mu-\nu) P^{n} \rightarrow 0$. We must show $\mu \sim \nu$. Let $\lambda_{0}=\mu \wedge \nu$, $r_{0}=\mu-\lambda_{0}, s_{0}=\nu-\lambda_{0}$. Let $\lambda_{n+1}=r_{n} P \wedge s_{n} P, r_{n+1}=r_{n} P-\lambda_{n+1}, s_{n+1}=$ $s_{n} P-\lambda_{n+1}$. Inductively define a randomized stopping time $\tau$ such that $Y_{k}$ has distribution $\lambda_{k}$ on $\{\tau=k\}$. Let $\lambda=\sum_{k=0}^{\infty} \lambda_{k}$. (See for example [4].) Since $r_{n} \rightarrow 0$ by assumption we have $\mu \rightarrow \lambda$ by $\tau$. Similarly $\nu \rightarrow \lambda$, so the theorem is proved.

The theorem of Ornstein and Sucheston [10] gives sufficient conditions for Theorem 3 to be applicable. For example, the operator studied in [2] (called $T$ in that paper) satisfies these conditions. The operator $P$ of $\$ 4$ of the present paper is a special case of the operator studied in [2].

Let $(S, \mathcal{G})$ be a measurable space. Let $p(x, A)$ be a Markov transition function on $S \times \mathcal{G}$, let $P$ be the associated Markov operator, and let $\left(Y_{n}\right)$ be the associated Markov process. Let $p(x, \cdot)$ be denoted by $\mu_{x}$. A function $h$ on $S$ will be called invariant if $P h$ exists and $P h=h$, that is,

$$
h(x)=\int h d \mu_{x} \text { for all } x \text { in } S .
$$


Let $\mathcal{H}$ denote a class of abstract harmonic functions. We assume nothing about $\mathcal{H}$ except that $\mathcal{H}$ is a subset of the invariant functions. Two bounded measures $\mu$ and $\nu$ on $\mathcal{G}$ will be said to be harmonically equivalent, $\mu \equiv \nu(\mathcal{H})$, if

$$
\int h d \mu=\int h d \nu \text { for all functions } h \text { in } \mathcal{H} \text {. }
$$

Clearly if $\mu \sim \nu$ in the sense of Definition 1, then $u \equiv \nu$ (bounded invariant functions).

Consider the following two statements:

Property 1. Any bounded invariant function is harmonic.

Property 2. $\mu \equiv \nu$ (bounded functions in $\mathcal{H}$ ) if and only if $\mu \sim \nu$.

We take Property 1 as the abstract form of the converse mean value theorem. Property 2 gives a characterization of asymptotic mass cancellation. We wish to give conditions under which the two properties are equivalent.

Assume that if $h$ is a bounded $\mathcal{G}$-measurable function such that $\int h d \mu=\int h d \nu$ whenever $\mu \equiv \nu$ then $h$ is harmonic.

Under assumption (2.3), which obviously holds in all the standard cases, Property 2 is easily seen to imply Property 1 . We now add further conditions:

Let there exist a sequence of sets $A(k)$ in $\mathcal{G}$ such that $A(k) \searrow \varnothing$, and such that $\left(Y_{n}\right)$ hits each $A(k)$ with probability 1 starting from any point.

Let $\left\{\mu_{x}: x\right.$ in $\left.S\right\}$ be separable with respect to the total variation norm.

For each $k$, let $P_{k}$ be the hitting operator of $A(k)$. That is, $\mu P_{k}$ is the distribution of $\left(Y_{n}\right)$ stopped at the first hitting time of $A(k)$ when the initial distribution is $\mu$.

We note that assumption (2.4) easily implies (by the strong Markov property) that

$$
\begin{aligned}
& \mu \sim \nu \text { if and only if }(\mu-\nu) P_{k} \rightarrow 0 \text { in total variation norm } \\
& \text { as } k \rightarrow \infty .
\end{aligned}
$$

TheOREM 4. Under assumptions (2.3)-(2.5), Properties 1 and 2 are equivalent.

Proof. We shall assume that Property 1 holds, and prove Property 2. As noted earlier, $\mu \sim \nu$ always implies $\mu \equiv \nu$. Thus if Property 2 does not hold, then there exists probability measures $\nu$ and $\lambda$ on $\mathcal{G}$ such that $\nu \equiv \lambda$ holds, but $\nu \sim \lambda$ does not hold.

Let $k(j)$ be a subsequence of the natural numbers, and $B(j)$ a sequence of measurable subsets of $A(k(j))$ such that $\mu_{x} P_{k(j)}(B(j))$ converges as $j \rightarrow \infty$ for 
each $x$ in $S$, and such that the sequences $\nu P_{k(j)}(B(j))$ and $\lambda P_{k(j)}(B(j))$ converge, as $j \rightarrow \infty$, to different limits.

Since $A(k) \searrow \varnothing$, it follows that for any bounded measure $\mu, \mu P_{k}-\mu P P_{k} \rightarrow$ 0 in total variation norm as $k \rightarrow \infty$. Hence $\mu P_{k(j)}(B(j))$ converges as $j \rightarrow \infty$ for every bounded measure $\mu$, and $\mu P P_{k(j)}(B(j))$ converges to the same limit.

Let $h(x)=\lim _{j \rightarrow \infty} \mu_{x} P_{k(j)}(B(j))$. Clearly $h$ is $\mathcal{G}$-measurable and $h=P h$, so $h$ is invariant, while $\int h d \nu \neq \int h d \lambda$, so $h$ is not harmonic. This contradiction proves Theorem 4.

Taking $\mathcal{H}=$ the invariant functions gives the following obvious corollary, which in turn easily implies the theorem.

CoROllaRY. $\mu \equiv \nu$ (bounded invariant functions) if and only if $\mu \sim \nu$.

Let us now add some further assumptions:

For any $x$ in $S$ and any fixed $k$, there exist a number $c$ and an integer $l>0$ such that $\mu_{y} \leqslant c \mu_{x} P^{l}$ for all $y$ in $S-A(k)$.

For any $x$ in $S$ and any integer $l \geqslant 0$, there is a $k$ with $\mu_{x} P^{l}(A(k))=0$.

A measure $\mu$ will be said to have compact support if $\mu(A(k))=0$ for some $k$. Assumption (2.8) says that each measure $\mu_{x} P^{l}$ has compact support. Assumption (2.7) implies among other things that any nonnegative invariant function which is finite at one point must be bounded on every set of the form $S-A(k)$, that is, locally bounded.

One more rather weak assumption will be made:

If $h$ is a nonnegative $\mathcal{G}$-measurable function such that $\int h d \mu$ $=\int h d \nu$ whenever $\mu \equiv \nu(\mathcal{H})$ and $\mu$ and $\nu$ have compact support then $h$ is harmonic.

Consider the following statements:

Property 3. Any finite nonnegative invariant function is harmonic.

Property 4. For any two measures $\mu$ and $\nu$ with compact support such that $\mu \equiv \nu(\mathcal{H})$, there exists a sequence of numbers $c(k) \rightarrow 1$, such that for all sufficiently large $k, \mu P_{k} / c(k) \leqslant \nu P_{k} \leqslant c(k) \mu P_{k}$.

Theorem 5. Under the preceding assumptions, Properties 3 and 4 are equivalent.

The proof of Theorem 5 is similar to that of Theorem 4, and is omitted.

All the assumptions of this section are true for the operator $P$ of $\S 4$. The assumptions prior to Theorem 4 hold for a wider class of operators, for example the operators of [2] and [11]. We shall show that the operator in $\$ 4$ has Property 4. 
In the context of Theorem 3 and the Corollary to Theorem 4 one should note the results of Foguel [6] for the case of convolutions on a group, which give more information in that case.

3. Modelling with Brownian motion. We require several facts for use in $\S 4$, beginning with the following, which is proved in [3]:

TheORem 6. Let $\left(\Omega, \mathcal{F}_{,} \mathcal{F}_{t}, X_{t}, \theta_{t}, \operatorname{Pr}_{x}\right)$ be standard Brownian motion on $\mathbf{R}^{l}$. Let $\mu$ and $\nu$ be probability measures on $\mathbf{R}^{l}$ such that $\operatorname{Pot} \nu$ is finite and continuous on $\mathbf{R}^{l}$, and $\operatorname{Pot} \mu \geqslant \operatorname{Pot} \nu$ on $\mathbf{R}^{l}$.

Then there exists an $\mathcal{F}_{t}$-stopping time $\tau$ such that $X_{\tau}$ has distribution $\nu$ with respect to $\operatorname{Pr}_{\mu}$.

It should be noted that Theorem 6 is proved in [3] under a slightly stronger assumption on Pot $\mu$ and Pot $\nu$, in the case that $l=1$ or $l=2$. However, the same proof as in [3] gives Theorem 6 as stated here, if one employs a stonger form of the domination principle, appropriate to $\mathbf{R}^{\mathbf{1}}$ and $\mathbf{R}^{2}$. We omit these details since for all applications made in the present paper, either version of Theorem 6 would suffice. However, in order to generalize Theorem 6, we will outline part of a proof which differs slightly from that given in [3].

Domination Principle. Let $\mu, \nu$ be probability measures on $\mathbf{R}^{n}, n>1, c$ a number. Suppose Pot $\nu$ is finite everywhere and Pot $\mu+c>\operatorname{Pot} \nu$ on support $\nu$. Then Pot $\mu+c \geqslant$ Pot $\nu$ on $\mathbf{R}^{n}$.

Proof of Theorem 6. Let $C$ be a countable collection of balls forming a base for the topology of $\mathbf{R}^{l}$. Let $U_{n}$ be a sequence of elements in $\mathcal{C}$ such that every element of $\mathcal{C}$ occurs infinitely often in the sequence.

Let $\tau(0)=0$; having defined $\tau(n)$, let $\mu_{n}$ be the distribution of $X_{\tau(n)}$ with respect to $\operatorname{Pr}_{\mu}$. If there exists some constant $c$ with Pot $\mu_{n}>c>\operatorname{Pot} \nu$ on $U_{n+1}$, then let $\tau(n+1)$ be the first time of hitting $U_{n+1}^{c}$ after $\tau(n)$. Otherwise, let $\tau(n+1)=\tau(n)$.

\section{Let $\tau=\lim _{n \rightarrow \infty} \tau(n)$.}

Just as in [3], we find that $\tau<\infty$ holds $\operatorname{Pr}_{\mu}$-a.e. Let $\gamma$ be the distribution of $X_{\tau}$ with respect to $\operatorname{Pr}_{\mu}$. We must show that $\gamma=\nu$.

Let $G=\{x \mid \operatorname{Pot} \gamma(x)>$ Pot $\nu(x)\}$. Since Pot $\gamma$ is lower semicontinuous, $G$

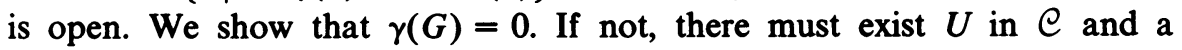
number $c$ such that Pot $\gamma>c>$ Pot $\nu$ on $U$, and $\gamma(U)>0$. Let $n(j)$ be a sequence with $U_{n(j)}=U$. Let $\lambda$ denote the measure that results from balayaging $\gamma$ onto $U^{c}$. Then $\gamma \neq \lambda$. But clearly $\mu_{n(j)} \Rightarrow \gamma$ and $\mu_{n(j)+1} \Rightarrow \lambda$. This contradicts $\mu_{n} \Rightarrow \gamma$, so we must conclude that $\gamma(G)=0$.

Once we know that $\gamma(G)=0$, the equality of Pot $\gamma$ and Pot $\nu$ follows from the domination principle. Hence $\gamma=\nu$, and Theorem 6 is proved.

The proof of Theorem 6 also shows: 
Corollary. Let $F=\{x$ : Pot $\mu(x)=$ Pot $\nu(x)\}$. The stopping time $\tau$ constructed in Theorem 6 is less than or equal to the first hitting time of $F$.

We now generalize Theorem 6 slightly:

TheORem 7. Let $\left(\Omega, \mathscr{F}_{,} \mathscr{F}_{t}, X_{t}, \theta_{t}, \operatorname{Pr}_{x}\right)$ be the standard Brownian motion on $\mathbf{R}^{l}$. Let $(\Gamma, \mathcal{G})$ be some measurable space. Let $\mathscr{B}$ be the Borel sets in $\mathbf{R}^{l}$. Let $\mu$ and $\nu$ be maps from $\Gamma \times \mathscr{B}$ to $\mathbf{R}$ such that $\mu(\alpha, \cdot), \nu(\alpha, \cdot)$ are probability measures on $\mathfrak{B}$ for each $\alpha$ in $\Gamma$ and $\mu(\cdot, A), \nu(\cdot, A)$ are $\mathcal{G}$-measurable functions for each $A$ in $\mathscr{B}$. Suppose $\operatorname{Pot} \nu(\alpha, \cdot)$ is finite and continuous on $\mathbf{R}^{l}$ and Pot $\mu(\alpha, \cdot)>\operatorname{Pot} \nu(\alpha, \cdot)$ on $\mathbf{R}^{l}$ for each $\alpha$ in $\Gamma$.

Then there exists $\tau: \Gamma \times \Omega \rightarrow[0, \infty]$ such that $\{\tau<t\}$ is in $\mathcal{G} \times \mathscr{F}_{t}$ for all $t$ and $X_{\tau(\alpha,)}$ has distribution $\nu(\alpha, \cdot)$ with respect to $\operatorname{Pr}_{\mu(\alpha,)}$ for each $\alpha$ in $\Gamma$.

To prove Theorem 7 one carries out the construction for $\tau$ just given in Theroem 6, using the same collection $\mathcal{C}$ for every $\alpha$. The measurability property of $\tau$ is easily verified.

TheOREM 8. Let $\left(\Omega, \mathscr{F}_{,} \mathscr{F}_{t}, X_{t}, \theta_{t}, \operatorname{Pr}_{x}\right)$ be standard Brownian motion on $\mathbf{R}^{l}$. Let $D$ be an open set in $\mathbf{R}^{l}$. Let $P$ be a Markov operator on $D$. Let $m_{x}$ denote the unit mass at $x$. Let $\lambda_{x}=m_{x} P$ for all $x$ in $D$. Suppose that Pot $\lambda_{x}$ is finite and continuous on $\mathbf{R}^{l}$ and Pot $m_{x}>\operatorname{Pot} \lambda_{x}$ on $\mathbf{R}^{l}$ for all $x$ in $D$. For each $x$ in $D$, suppose there exists an open set $V(x) \subseteq D$ such that Pot $m_{x}=$ Pot $\lambda_{x}$ outside $V(x)$. Then there exists a sequence of stopping times $T(n)$ such that if $Y_{n}=$ $X_{T(n)}$ and $\mathcal{G}_{n}=\mathscr{F}_{T(n)}$ then $\left(\Omega, \mathscr{F}_{,} \mathcal{G}_{n}, Y_{n}, \operatorname{Pr}_{x}\right), x$ in $D$, is a Markov process with operator $P$. Furthermore, each $T(n)$ is less than or equal to the first exit time of $D$, and for any $\omega, X_{t}(\omega)$ is in $V\left(Y_{n}(\omega)\right)$ for $T(n)<t<T(n+1)$.

Proof. In Theorem 4, let $\Gamma=D$, let $\mathcal{G}$ be the Borel subsets of $D$, let $\mu(x, \cdot)=m_{x}, \nu(x, \cdot)=\lambda_{x}$. Let $\tau$ be the corresponding map on $D \times \Omega$. By the corollary to Theorem $6, \tau(x, \cdot)$ is less than or equal to the first exit time of $V(x)$. Set $T(0)=0, T(n+1)=\tau\left(X_{T(n)}, \cdot\right)+T(n)$. The measurability property of $\tau$ easily implies that each $T(n)$ is a stopping time. The fact that $\left(Y_{n}\right)$ is a Markov process then follows from the strong Markov property of the $\left(X_{t}\right)$ process. The remaining properties of the $T(n)$ follow at once from the bound on $\tau$, so Theorem 8 is proved.

Strictly speaking one should add a cemetery point $\Delta$ to the state space of the $\left(X_{t}\right)$ process, and set $\tau(\Delta, \cdot)=0$, in order to take care of those $\omega$ with $T(n)=\infty$ for some $n$. Alternatively, one could discard such sample paths, since they form a null set with resepct to all probabilities $\operatorname{Pr}_{x}$.

For other results on modelling processes with Brownian motion, see [5] and [8].

4. Proof of Theorem 2. The function $\varphi$ of $\S 1$ is the kernel for a Markov operator $P$. Let $\varphi_{n}$ be the corresponding kernel for $P^{n}$. The following simple 
"local cancellation" lemma is similar to a lemma in [2].

LEMMA 1. There exist a number $c>0$ and an integer $N$ such that if $x, y, z$ are in $D$ and $|y-x| \leqslant(1-\alpha) d(x),|z-x|\left\langle(1-\alpha) d(x)\right.$ then $\left.\varphi_{N}(y, \cdot)\right\rangle$ $c \varphi(z, \cdot)$ on $\mathbf{R}^{l}$.

Proof. Using compactness and scaling, it is easy to see that there exists a constant $\zeta$ depending only on $\alpha$ such that if $x, y, w$ are in $\mathbf{R}^{l}$ with $|x-y|<$ $(1-\alpha) r,|y-w| \leqslant\left(\operatorname{tr}+\alpha^{2} r / 2\right),|x-w|<(1-\alpha) r, t>\alpha^{2} / 2$, then the volume of $B(y, \operatorname{tr}) \cap B(x,(1-\alpha) r) \cap B\left(w, \alpha^{2} r\right)$ is at least $\zeta r^{l}$.

Let $r=d(x)$. Then $d(v) \geqslant \alpha r$ for all $v$ with $|x-v|<(1-\alpha) r$.

It follows from (1.2) that there exists a constant $\xi$, depending only on $\alpha$ and $\beta$, such that $\varphi(v, \cdot) \geqslant \xi r^{-l}$ on $B\left(v, \alpha^{2} r\right)$ for all $v$ with $|x-v|<(1-\alpha) r$.

By induction,

$$
\varphi_{n}(v, \cdot) \geqslant \xi^{n \zeta^{n-1}} r^{-l} \text { on } B\left(v, n \alpha^{2} r / 2\right) \cap B(x,(1-\alpha) r)
$$

for all $v$ with $|x-v| \leqslant(1-\alpha) r$.

Fix $n$ larger than $4 / \alpha^{2}$. Then $\varphi_{n}(y, \cdot) \geqslant \xi^{n \xi^{n-1}} r^{-l}$ on $B(x,(1-\alpha) r)$.

Let $\rho=d(z)$. Clearly there exists a constant $\lambda$ depending only on $\alpha$ such that $B(x,(1-\alpha) r) \cap B(z,(1-\alpha) \rho)$ has volume at least $\lambda r^{l}$.

By the previous argument, with $x$ replaced by $z$ and $r$ replaced by $\rho$, $\varphi_{n}(v, \cdot) \geqslant \xi^{n} \xi^{n-1} \rho^{-l}$ on $B(z,(1-\alpha) \rho)$, for all $v$ in $B(z,(1-\alpha) \rho)$.

Hence

$$
\varphi_{2 n}(y, \cdot) \geqslant \xi^{2 n} \zeta^{2 n-2} \lambda \rho^{-l} \text { on } B(z,(1-\alpha) \rho) .
$$

By (1.2), there exists a constant $\psi$ depending only on $\alpha$ and $\gamma$ such that $\varphi(z, \cdot)<\psi \rho^{-l}$ on $B(z,(1-\alpha) \rho)$. Since $\varphi(z, \cdot)=0$ outside $B(z,(1-\alpha) \rho)$, we have $\varphi_{2 n}(y, \cdot) \geqslant\left(\xi^{\left.2 n \xi^{2 n-2} \lambda / \psi\right) \varphi(z, \cdot)}\right.$ on $\mathbf{R}^{l}$. Taking $N=2 n$ and $c=$ $\xi^{2 n} \zeta^{2 n-2} \lambda / \psi$, the lemma is proved.

Let $\varphi$ satisfy conditions (1.1)-(1.3). Let $\mu_{x}$ be defined as in $\S 1$. Let $P$ be the Markov operator on $D$ with transition density $\varphi$, so that $m_{x} P=\mu_{x}$ for all $x$ in $D$. By Theorem 8 of $\S 3$, there exist stopping times $T(n)$ for Brownian motion $\left(\Omega, \mathscr{F}_{1}, \mathscr{F}_{t}, X_{t}, \theta_{t}, \operatorname{Pr}_{x}\right)$ such that if $Y_{n}=X_{T(n)}, \mathcal{G}_{n}=\mathscr{F}_{T(n)}$ then $\left(\Omega, \mathscr{F}, \mathcal{G}_{n}, Y_{n}, \operatorname{Pr}_{x}\right), x$ in $D$, is a Markov process with operator $P$. Furthermore, $T(n)$ is less than or equal to the first exit time of $D$ for each $n$, and for any $\omega, X_{t}(\omega)$ is in $B\left(Y_{n}(\omega),(1-\alpha) d\left(Y_{n}(\omega)\right)\right)$ for $T(n) \leqslant t<T(n+1)$.

Let $A(k)$ be a sequence of open subsets of $D$, such that $A(k) \backslash \varnothing, \overline{A(k+1)}$ $\subseteq A(k), D-A(k)$ is compact for each $k$. As in $\$ 2$, let the hitting operator of $A(k)$ using $P$ be denoted by $P_{k}$. It is easy to see that the Markov process $\left(Y_{n}\right)$ hits each $A(k)$ with probability 1 , starting from any point in $D$ (cf. [2, §3]).

Lemma 2. Let $\mu$ and $\nu$ be two probability measures with compact support in $D$ such that Pot $\mu=$ Pot $\nu$ outside some compact subset of $D$. Let $c$ be the constant in Lemma 1. Then for some $k, \mu P_{k} \geqslant c \nu P_{k}$. 
Proof. Pot $\mu=$ Pot $\nu$ on some $A(l)$. By (1.3), Pot $\nu \geqslant$ Pot $\nu P_{l}$. Replacing $\nu$ by $\nu P_{l}$ if necessary, we may assume that Pot $\mu \geqslant$ Pot $\nu$ on the support of $\nu$, and hence, by the domination principle, on $\mathbf{R}^{l}$. By Theorem 6 , there exists a stopping time $\sigma$ for Brownian motion, such that $X_{\sigma}$ has distribution $\nu$ with respect to $\operatorname{Pr}_{\mu}$. The corollary to Theorem 6 shows that $\sigma$ may be chosen less than or equal to the first exit time of some compact subset of $D$. Let $U=\inf \{n: T(n) \geqslant \sigma\}$. It is easy to see that $U$ is a $\mathcal{G}_{n}$-stopping time. It is clear that $U$ is less than or equal to the first hitting time of some $A(l)$. For any fixed $\omega$, let $U(\omega)=n, \sigma(\omega)=t$. Let $x=Y_{n-1}(\omega), y=Y_{n}(\omega), z=X_{t}(\omega)$. Then $|y-x| \leqslant(1-\alpha) d(x)$ and $|z-x| \leqslant(1-\alpha) d(x)$. By Lemma $1, \varphi_{N}(y, \cdot) \geqslant$ $c \varphi(z, \cdot)$ on $\mathbf{R}^{l}$. Hence $\varphi_{N}\left(Y_{U}, \cdot\right) \geqslant c \varphi\left(X_{\sigma}, \cdot\right)$ on $\mathbf{R}^{l}$ for all $\omega$. Let $\bar{\mu}$ be the distribution of $Y_{U+N}$ with respect to $\operatorname{Pr}_{\mu}$. Then $E_{\mu} \varphi_{N}\left(Y_{U}, \cdot\right) \geqslant c E_{\mu} \varphi\left(X_{\sigma}, \cdot\right)$ on $\mathbf{R}^{l}$ gives $\bar{\mu} \geqslant c \nu P$. Let $k$ be chosen such that $U+N$ is less than or equal to the first hitting time of $A(k)$ with $\operatorname{Pr}_{\mu}$-probability 1 , and such that $\nu(A(k))=$ 0 . Then $\mu P_{k}=\bar{\mu} P_{k}$, and $\nu P_{k}=\nu P P_{k}$. Thus $\mu P_{k} \geqslant c \nu P_{k}$, so Lemma 2 is proved.

LEMMA 3. Let $\mu$ and $\nu$ be two probability measures with compact support in $D$ such that Pot $\mu=\operatorname{Pot} \nu$ outside some compact subset of $D$. Then for every $j$ there exists an $n$ such that

$$
\mu P_{n} \geqslant c \sum_{i=0}^{j-1}(1-c)^{i} \nu P_{n}
$$

Proof. By induction. Lemma 2 gives the result for $j=1$. Suppose the result holds for some $j$ and $n$. Let

$$
w=c \sum_{i=0}^{j-1}(1-c)^{i} \nu P_{n} .
$$

Let $u=\mu P_{n}-w, v=\nu P_{n}-w$. Then $v=(1-c)^{j} \nu P_{n}$. After normalizing, $u$ and $v$ satisfy conditions of Lemma 2 , so a $k \geqslant n$ may be chosen with $u P_{k} \geqslant c v P_{k}$. Then

$$
\begin{aligned}
\mu P_{k} & =\mu P_{n} P_{k}=w P_{k}+u P_{k} \geqslant w P_{k}+c v P_{k} \\
& =c \sum_{i=0}^{j}(1-c)^{i} \nu P_{n} P_{k}+c(1-c)^{j} \nu P_{n} P_{k}=c \sum_{i=0}^{j+1}(1-c)^{i} \nu P_{k} .
\end{aligned}
$$

This proves Lemma 3.

We note that Lemma 3 implies that $P$ has Property 4 of $\$ 2$.

Proof of Theorem 2. Let $h$ be a finite, nonnegative, Lebesgue measurable invariant function. Let $x$ be any point in $D$. Let $\mu$ and $\nu$ be two probability measures, each having uniform density on some ball in $D$ with centre $x$. We shall show that $\int h d \mu=\int h d \nu$, so that $h$ has the ordinary mean value property. It then follows at once that $h$ is harmonic. To prove that $\int h d \mu=$ 
$\int h d \nu$, we note first that any invariant function $h$ is easily seen to be bounded on compact subsets of $D$. Hence for any $n, \int h d \mu=\int h d\left(\mu P_{n}\right)$ and $\int h d \nu=$ $\int h d\left(\nu P_{n}\right)$. From Lemma 3 , for any $j$ we see that

$$
\int h d \mu \geqslant c \sum_{i=0}^{j-1}(1-c)^{i} \int h d \nu .
$$

Hence $\int h d \mu>\int h d \nu$. By symmetry $\int h d \mu \leqslant \int h d \nu$, so the theorem is proved.

\section{REFERENCES}

1. M. A. Akcoglu and R. W. Sharpe, Ergodic theory and boundaries, Trans. Amer. Math. Soc. 132 (1968), 447-460.

2. J. R. Baxter, Restricted mean values and harmonic functions, Trans. Amer. Math. Soc. 167 (1972), 451-463.

3. J. R. Baxter and R. V. Chacon, Potentials of stopped distributions, Illinois J. Math. 18 (1974), 649-656.

4. __ Stopping times for recurrent Markov processes, Illinois J. Math. 20 (1976), 467-475.

5. R. V. Chacon, Potential processes, Trans. Amer. Math. Soc. 226 (1977), 39-58.

6. S. R. Foguel, Iterates of a convolution on a non-Abelian group, Ann. Inst. H. Poincaré Sect. B (N. S.) 11 (1975), 199-202.

7. D. Heath, Functions possessing restricted mean value properties, Proc. Amer. Math. Soc. 41 (1973), 588-595.

8. I. Monroe, On embedding right continuous martingales in Brownian motion, Ann. Math. Statist. 43 (1972), 1293-1311.

9. S. Orey, An ergodic theorem for Markov chains, Z. Wahrscheinlichkeitstheorie und Verw. Gebiete 1 (1962), 174-176.

10. D. S. Ornstein and L. Sucheston, An operator theorem on $L_{1}$ convergence to zero with application to Markov kernels, Ann. Math. Statist. 41 (1970), 1631-1639.

11. W. A. Veech, A zero-one law for a class of random walks and a converse to Gauss' mean value theorem, Ann. of Math. (2) 97 (1973), 189-216.

12. _ A converse to the mean value theorem for harmonic functions, Amer. J. Math. 97 (1975), 1007-1027.

13. _ The core of a measurable set and a problem in potential theory (preprint).

Department of Mathematics, University of Minnesota, Minneapolis, Minnesota 55455 